\title{
A SIEGEL FORMULA FOR ORTHOGONAL GROUPS OVER A FUNCTION FIELD
}

\author{
BY
}

\author{
STEPHEN J. HARIS ( 1 )
}

ABSTRACT. We obtain a Siegel formula for a quadratic form over a function field, by establishing the convergence of the corresponding Eisenstein-Siegel series directly, then via the Hasse principle, that of the associated Poisson formula.

Introduction. In this paper, we obtain a Siegel formula, as recast by Weil [7], for a quadratic form over a function field. The difficulty is that there is no criterion to guarantee the convergence of the integral

$$
\int_{G_{A} / G_{k}} \sum_{\xi \in X_{k}} \Phi(g \cdot \xi)|d g|_{A},
$$

which occurs in the formula (see $\$ 1$ for the notation), as was the case for $k$ a number field, cf. Weil [7], Igusa [2]. We establish convergence of the corresponding Siegel-Eisenstein series, then by the Hasse principle obtain the Siegel formula and the convergence of the above integral.

The author wishes to acknowledge his thanks to Professor Igusa for numerous helpful conversations.

1. Notation and the Siegel formula. Let $k$ be a function field in one variable over a finite constant field, that is, a finitely generated extension of a finite prime field $F_{q}$, of degree of transcendence one over $F_{q}$. We. shall assume that characteristic $(k) \neq 2$.

Let $X$ be a vector space of dimension $m$ and $q(x)$ a nondegenerate quadratic form on $X$, all defined over $k$. Take $G=S O(q)$ (a semisimple algebraic group, defined over $k$, for $m \geq 3$ ) to be the special orthogonal group of $q$. The Siegel formula is given for the standard representaion $\rho: G \rightarrow$ Aut $(X)$; it reads

$$
\int_{G_{A} / G_{k}}\left(\sum_{\xi \in X_{k}} \Phi(g \cdot \xi)\right)|d g|_{A}=2 \sum_{i^{*} \in k} \int_{X_{A}} \Phi(x) \chi\left(q(x) i^{*}\right)|d x|_{A}+2 \Phi(0)
$$

Received by the editors January 17, 1973.

AMS (MOS) subject classifications (1970). Primary 10C05, 10C30; Secondary 20G30, $12 A 90$.

(1) Partial support from NSF grant GP $31820 X$. 
where $G_{A}, G_{k}$ are the adelisation, the k-rational points (respectively) of $G$; $\Phi \in \mathcal{S}\left(X_{A}\right)$ is a Schwartz function on the adelisation of $X$ and $X$ is a fixed, nontrivial character of $k_{A}$, the adelisation of $k$, which is 1 on $k$.

2. Orbits, stablisers. To analyse the integral $\int_{G_{A} / G_{k}} \Sigma_{\xi \in X_{k}} \Phi(g \cdot \xi)|d g|_{A}$, we recall results established by Weil $[7, \S 14-29]$. The orbits of $G$ in $X$ which contain points of $X_{k}$ are the sets $U(i)=\{x \in X \mid q(x)=i, x \neq 0\}$ where $i \in k$, $U(i)_{k} \neq \varnothing$, and $\{0\}$. 'This is precisely Witt's theorem. Further, two points $x, y \in$ $X_{K}$, not zero, belong to the same orbit of $G_{K}$ if and only if they belong to the same orbit of $G$. This for any $K \supset k$.

For the nonempty $U(i)_{k}$, fix $\xi_{i} \in U(i)_{k}$ and let $H_{i}$ be the stabiliser of $G$ at $\xi_{i}$, i.e., $H_{i}=\left\{g \in G \mid g \cdot \xi_{i}=\xi_{i}\right\}$, an algebraic group defined over $k$. Hence

$H_{i}=S O(m-1)$, of rank $m-1$, for $i \neq 0$;

$H_{0}=S O(m-1) \cdot$ unipotent, a semidirect product;

and in all cases, the Tamagawa numbers for $G, H_{i}$ are 2, Weil [5].

Furthermore, the mapping $g \rightarrow g \cdot \xi_{i}$ of $G \rightarrow U(i)$ induces an isomorphism of $G / H_{i}$ onto $U(i)$. By Witt's theorem there is a generic section for this map. Also, as $k$ is an infinite field and $G$ is a reductive group, $G_{k}$ is Zariski dense in $G$ (Borel [1]). Whence, the mapping $g \rightarrow g \cdot \xi_{i}$ inducesathe identification $G_{A} /\left(H_{i}\right)_{A}=U(i)_{A}$ of the adelisations.

Take $\Phi \in \mathcal{S}\left(X_{A}\right)$; then for $i \in k$ so that $U(i)_{k} \neq \varnothing$,

$$
\int_{G_{A} / G_{K}} \sum_{\xi \in U(i)_{k}} \Phi(g \cdot \xi)|d g|_{A}=\tau\left(H_{i}\right) \int_{U(i)_{A}} \Phi\left|D_{i}\right|_{A},
$$

where $r\left(H_{i}\right)$ is the Tamagawa number of $H_{i},\left|D_{i}\right|_{A}$ is the Tamagawa measure derived from $D_{i}=d g / d b_{i}, d g, d b_{i}$ invariant differential forms of maximal degree without zeros or poles for $G, H_{i}$, respectively, defined over $k$. The convergence factors may be taken to be 1 , from the explicit nature of the stabilisers $\boldsymbol{H}_{\boldsymbol{i}}$ •

By the Hasse principle for quadratic forms, $U(i)_{k}=\varnothing$ implies that $U(i)_{A}=$ $\varnothing$. Thus we see that (1) is valid for all $i \in k$.

3. Asymptotic estimates. Let $v$ be a valuation on $k$, which is trivial on the field of constants, with $k_{v}$ as the completion. Then $k_{v}$ is nonarchimedean and denote by $\mathcal{O}_{v}, p_{v}$ and $q_{v}$ the maximal compact subring of $k_{v}$, the ideal of nonunits of $\mathcal{O}_{v}$ and the number of elements of $\mathcal{C}_{v} / p_{v}$ (resp.). Let $X_{v}, X_{v}^{0}$ be the $k_{v}, \mathcal{O}_{v}$ (resp.) rational points of $X$ and $|d i|_{v},|d x|_{v}$ be autodual measures on $k_{v}$ and $X_{v}$.

For $X_{v}$ a nontrivial character of $k_{v}$, we identify $X_{v}$ with its dual by $\left(x, x^{\prime}\right)$ $\rightarrow X_{v}\left(x^{t} x^{\prime}\right)$, where we write the elements of $X_{v}$ as row vectors, with respect to some $k$-basis. For $\Phi \in \delta\left(X_{v}\right)$, the Schwartz-Bruhat space, the Fourier transform 
is defined by $\Phi^{*}\left(x^{*}\right)=\int_{X_{v}} \Phi(x)\left\langle x, x^{*}\right\rangle|d x|_{v}$, where $\left\langle x, x^{*}\right\rangle=x^{*}(x)$. We choose as before $|d x|_{v}$ to be the autodual measure on $X_{v}$.

For $\Phi \in S\left(X_{v}\right)$, we consider the function for $i^{*} \in k_{v}$ defined by

$$
F_{\phi}^{*}\left(i^{*}\right)=\int_{X_{v}} \Phi(x) \chi_{\nu}\left(q(x)_{i}^{*}\right)|d x|_{\nu}
$$

The first sections of Weil [7] are devoted to proving general properties of such functions, in actually a more general setting. Namely, for $X, Y$ locally compact abelian groups and $f: X \rightarrow Y$ a continuous mapping, the principal result concerns the decomposition of the measure $d x$ on $X$, when $f$ satisfies a "condition (A)". If $\Lambda(X)$ denotes the subspace of $\mathscr{L}^{1}(X)$ consisting of those continuous functions $\Phi$ with $\Phi^{*} \in \mathfrak{L}^{1}\left(X^{*}\right)$, then Fourier transformation gives a bijection of $\Lambda(X)$ with $\Lambda\left(X^{*}\right)$, so that $\left(\Phi^{*}\right)^{*}(x)=\Phi(-x)$ for every $x \in X$. Among other things, Weil proves that if $f$ satisfies "condition (A)", i.e.,

$$
F_{\phi}^{*}\left(y^{*}\right)=\int_{X} \Phi(x)\left\langle f(x), y^{*}\right\rangle d x
$$

is integrable on $Y^{*}$, uniformly so in $\Phi$ when $\Phi$ is restricted to a compact subset of $S(X)$, then

(i) $F_{\phi}^{*}$ belongs to $\Lambda\left(Y^{*}\right)$, and

(ii) there exists a unique family of measures $d \mu_{y}$ on $X$, each $d \mu_{y}$ being the image measure under $f^{-1}(y) \rightarrow X$, of a measure on $f^{-1}(y)$, such that $F_{\phi}^{*}$ becomes the Fourier transform of $F_{\Phi}(y)=\int_{X} \Phi(x) d \mu_{y}(x)$.

We shall show that in the local and global cases, $f=q$, the quadratic form satisfies "condition (A)".

A fact which will play an important role is that if $\psi: k_{v}^{n} \rightarrow \mathrm{T}$ is a nondegenerate second degree character of $k_{\nu}^{n}$, i.e., $\psi$ is continuous and satisfies $\psi(x+y)=\psi(x) \cdot \psi(y) \cdot\langle x, y b\rangle$ for some bicontinuous isomorphism $b: k_{v}^{n} \rightarrow\left(k_{v}^{n}\right)^{*}$, then its Fourier transform is given by

$$
\psi^{*}\left(x^{*}\right)=\gamma(\psi)|b|^{-1 / 2} \psi\left(x^{*} b^{-1}\right)^{-1},
$$

where $\gamma(\psi) \in \mathrm{T}$, a complex number of absolute value 1 , and $|b|$ is the modulus of $b$ (Weil [6, p. 161]). Hence

$$
\left.\left.\left|\int_{k_{v}^{n}} \Phi(z) \psi(z)\right| d z\right|_{\nu}\left|\leq\left\|\Phi^{*}\right\|_{1}\right| \operatorname{det} b\right|_{\nu} ^{-1 / 2} .
$$


For our case, take $\psi(x)=\chi_{v}(q(x))$, so that (2) reads $\left|F_{\Phi}^{*}\left(i^{*}\right)\right| \leq\left\|\Phi^{*}\right\|_{1}\left|i^{*}\right|_{v}^{-m / 2}$. Since, trivially, $\left|F_{\Phi}^{*}\left(i^{*}\right)\right| \leq\|\Phi\|_{1}$, we have

$$
\left|F_{\Phi}^{*}\left(i^{*}\right)\right| \leq \max \left(\|\Phi\|_{1},\left\|\Phi^{*}\right\|_{1}\right) \cdot \max \left(1,\left|i^{*}\right|_{\nu}\right)^{-m / 2} \text {. }
$$

Therefore, we have proved:

Lemma 1. Let $C$ be a compact subset of $S\left(X_{v}\right)$. Then, there exists a positive constant $c_{0}$ sucb that

$$
\left|F_{\Phi}^{*}\left(i^{*}\right)\right| \leq c \max \left(1,\left|i^{*}\right|_{v}\right)^{-m / 2}
$$

for all $\Phi \in C, i^{*} \in k_{\nu}$.

It is easy to che'ck that, for $t \in k_{v}^{\times}$,

$$
\int_{k_{v}} \max \left(|t|_{v},|i|_{v}\right)^{-\sigma}|d i|_{v}=\text { const }|t|_{v}^{1-\sigma}
$$

This, combined with Lemma 1, shows that $q: X_{v} \rightarrow k_{v}$ satisfies "condition (A)". Therefore, there exists a uniquely determined family of positive measures $\left\{\mu_{i} \mid i \in k_{v}\right\}$ on $X_{v}$, such that

(i) support $\left(\mu_{i}\right) \subset\left\{x \in X_{v} \mid q(x)=i\right\}$;

(ii) for any coutinuous function $\Phi$ with compact support on $X_{v}$, the function $F_{\Phi}(i)=\int_{X_{v}} \Phi(x) d \mu_{i}(x)$ defined on $k_{\nu}$ is continuous and satisfies

$$
\int_{k_{v}} F_{\phi}|d i|_{v}=\int_{X} \Phi(x)|d x|_{v}
$$

Moreover,

(iii) if $\Phi \in \mathcal{S}\left(x_{v}\right), F_{\Phi}$ is continuous, integrable and has as its Fourier transform

$$
F_{\phi}^{*}\left(i^{*}\right)=\int_{X_{\nu}} \Phi(x) \chi_{\nu}\left(q(x) i^{*}\right)|d x|_{\nu} \quad\left(i^{*} \in k_{\nu}\right)
$$

As the sets $U_{v}(i)=\left\{x \in X_{v} \mid q(x)=i, x \neq 0\right\}$ are in fact the fibres, for $i \neq 0$, these sets carry the measure $\mu_{i}$. But the same is true for $i=0$ 。 To see this, use $\Phi(t x)$ in place of $\Phi(x)$, for $t \in k_{v}^{x}$. The uniqueness of the measures implies that $\mu_{0}(t x)=|t|_{v}^{m-2} \mu_{0}(x)$, so that no part of the measure $\mu_{0}$ is carried by the set $\{0\}$.

To identify the measures $\mu_{i}$, consider the gauge form $D_{v_{0} i}(x)=(d x / d q(x))_{i}$ on $U_{v}(i)$. As $q$ is submersive on $X_{v}-\{0\}$, this is well defined and satisfies 


$$
\int_{X_{v}-\{0\}} \Phi|d x|_{v}=\int_{k_{v}}|d i|_{v} \int_{U_{v}(i)} \Phi\left|D_{v, i}\right|_{v}
$$

where $\left|D_{v, i}\right|_{v}$ is the measure on $U_{v}(i)$ determined by $D_{v_{0},}$. This holds for all continuous functions $\Phi$ with compact support contained in $X_{v}-\{0\}$. But $\{0\}$ has measure zero for $|d x|_{v}$, so we can extend the above equality to:

$$
\int_{X_{v}} \Phi|d x|_{v}=\int_{k_{v}}|d i|_{v} \int_{U_{v}(i)} \Phi\left|D_{v, i}\right|_{v}
$$

whence by the uniqueness of the family $\left\{\mu_{i}\right\}$, we have $\mu_{i}=\left|D_{\nu, i}\right|_{v}\left(i \in k_{v}\right)$.

It is convenient at this time to mention that the gauge form $D_{i}(x)=(d x / d q(x))_{i}$ on $U(i)$, for $i \in k$. is also defined and is invariant under $G$, so it differs from the earlier $d g / d b_{i}$ by a factor of $k^{\times}$. Thus the measures given by $D_{i}(x)$ and $d g / d b_{i}$ are the same, since the product formula is valid for $k^{\times}$.

Note that in the estimate (3), for $\Phi, \Phi^{*}$ the characteristic functions of $X_{v^{0}}^{0}$, $X_{v}^{0 *}$ we have $\left|F_{\phi}^{*}\left(i^{*}\right)\right| \leq \max \left(1,\left|i^{*}\right|_{v}\right)^{-m / 2}$.

4. A dominant series. We shall now prove the convergence of the SiegelEisenstein series. The method of proof is based on the following lemma and the methods used in [3], due to Igusa.

As always $k$ denotes a function field of transcendence degree one over a finite field $k_{0}$. We may assume that $k_{0}$ is algebraically closed in $k_{\text {. Put }} q=$ $\operatorname{card}\left(k_{0}\right)$ and let $g$ denote the genus of $k$. Choose a prime divisor $P_{\infty}$ of $k$ such that $d=\operatorname{deg}\left(P_{\infty}\right) \geq 2 g+1$, whence $l\left(P_{\infty}\right)=d+1-g \geq g+2$. So, there exists $x \in k$ with $(x)_{\infty}=P_{\infty}$.

Denote by $\mathcal{E}$ the $k$-normalization of $k_{0}[x]$. The group of units of $\mathcal{O}=k_{0}^{\times}$, hence finite. Also, every $b \neq 0 \in \mathcal{O}$ has $|b|_{\infty} \geq 1$.

Lemma 2. Let $\lambda$, a denote real numbers, $\lambda \geq 1, a>1$. Then

$$
\sum_{a \in \mathcal{E}} \max \left(\lambda,|a|_{\infty}\right)^{-a} \leq c \lambda^{1-a}
$$

where $c$ is independent of $\lambda$.

Proof. We have

$$
\sum_{a \in \theta} \max \left(\lambda,|a|_{\infty}\right)-^{a}=\sum_{e=0}^{\infty} \operatorname{card}\left(L\left(P_{\infty}^{e}\right)-L\left(p_{\infty}^{e-1}\right)\right) \max \left(\lambda, q^{d e}\right)^{-a} .
$$

Write $\lambda=q^{d \delta}$, so that $0 \leq[\delta] \leq \delta<[\delta]+1$. So 
where

$$
\sum_{a \in \theta} \max \left(\lambda,|a|_{\infty}\right)^{-a}= \begin{cases}A & \text { if }[\delta] \geq 1, \\ B & \text { if }[\delta]=0\end{cases}
$$

$$
\begin{aligned}
& A=\operatorname{card}\left(L\left(p_{\infty}^{[\delta]}\right)\right) \lambda^{-a}+\sum_{e=[\delta]+1}^{\infty}\left(q^{d e+1-8}-q^{d(e-1)+1-8}\right) q^{-d e a}, \\
& B=q \lambda^{-\alpha}+\left(q^{d+1-8}-q\right) q^{-d a}+\sum_{e=2}^{\infty}\left(q^{d e+1-8}-q^{d(e-1)+1-8}\right) q^{-d e a} .
\end{aligned}
$$

So, setting $\langle\delta\rangle=\delta-[\delta]$,

$$
\begin{aligned}
& A=\lambda^{1-\alpha}\left\{q^{1-8-d\langle\delta\rangle}+\frac{q^{1-8\left(1-q^{-d}\right) q^{-(a-1) d(1-\langle\delta\rangle)}}}{1-q^{-(a-1) d}}\right\} \\
& B=\lambda^{1-a}\left\{q^{1-d\langle\delta\rangle}+q^{-(a-1) d\langle\delta\rangle}\left[\left(q^{d+1-8}-q\right) q^{-d a}+\frac{q^{1-g\left(1-q^{-d}\right) q^{-2(a-1) d}}}{1-q^{-(a-1) d}}\right]\right\} .
\end{aligned}
$$

Fix this choice of generator $x$. The ideal class group of $k$ for this $\mathcal{Q}$ is finite and let $r_{1}, \cdots, r_{b}$ be coset representatives, which may be taken to be integral ideals. Set $S_{\infty}=\left\{P_{\infty}\right\}$.

Proposition 1. Let $n$ be a given integer $>0$, and $\epsilon>0$ be fixed. Suppose that for eacb valuation $v$ on $k, \sigma_{v}$ is a given real number, sucb that $\sigma_{v}>n$, for all $v, \sigma_{v} \geq n+1+\epsilon$, for almost all $v$. Then

is convergent.

$$
\sum_{i=\left(i_{1}, \cdots, i_{n}\right) \in k^{n}} \prod_{v} \max \left(1,\left|i_{1}\right|_{v}, \cdots,\left|i_{n}\right|_{\nu}\right)^{-\sigma_{v}}
$$

Proof. The convergence is clear for $n=0$, so suppose $n \geq 1$ and use induction. Let $E \subset\{1,2, \cdots, n\}$ be a subset and

$$
k_{E}=\left\{i \in k^{n} \mid i_{p} \neq 0 \text { for } p \in E, i_{p}=0 \text { for } p \notin E\right\} \text {. }
$$

Then we have the disjoint union $k^{n}=\bigcup_{E} k_{E}$. By induction, the partial sums over $k_{E}$ are convergent for every $E \neq\{1,2, \cdots, n\}$. So it remains to show that the partial sum over $\left(k^{\times}\right)^{n}$ is convergent.

By hypothesis, there is a finite set of valuations $S$ on $k, S \supset S_{\infty}$ such that $\sigma_{v}>n$ for all $v$ and $\sigma_{v} \geq \beta=1+n+\epsilon$ for all $v \notin S$. We can enlarye $S$ without changing $\beta$, so suppose $S$ contains all the prime factors of $r_{1}, \cdots, r_{b}$. Further, as a function of $\sigma, \max \left(1,\left|i_{1}\right|_{v}, \ldots,\left|i_{n}\right|_{v}\right)^{-\sigma}$ is monotone decreasing, so it suffices to prove convergence when. $\sigma_{v}=\alpha>_{n}, v \in S, \sigma_{v}=\beta, v \notin S$. 
Let $i=\left(i_{1}, \ldots, i_{n}\right) \in\left(k^{\times}\right)^{n}$. Then $i_{p} \mathcal{C}=\mathbf{a}_{p^{\prime}} \mathbf{b}$ for integral ideals $\mathbf{b}, \mathbf{a}_{1}, \ldots$, $a_{n}$. Choosing them to be relatively prime, this set is uniquely determined by $i$. Moreover, there is a unique index $j$ so that $b_{j}=\Theta_{b}$, for some $b \neq 0 \in \mathcal{C}$. Setting $a_{p}=b i_{p}$ we have $a_{p} \mathcal{C}=b_{j} i_{p}=a_{p} r_{j} \subset \mathcal{O}$ so $a_{p} \neq 0 \in \mathcal{C}$. By the choice of $S$

$$
\begin{aligned}
\prod_{v} \max \left(1,\left|i_{1}\right|_{v}, \cdots,\left|i_{n}\right|_{v}\right)^{-\sigma}= & \prod_{v \in S}|b|_{v}^{a} \max \left(|b|_{v},\left|a_{1}\right|_{v}, \cdots,\left|a_{n}\right|_{v}\right)^{-a} \\
& \times \prod_{v \notin S}|b|_{v}^{\beta} \max \left(|b|_{v},\left|a_{1}\right|_{v}, \cdots,\left|a_{n}\right|_{v}\right)^{-\beta} .
\end{aligned}
$$

But, as the prime factors of the $\mathbf{r}_{j}$ are in $S$, max $v\left(|b|_{v},\left|a_{1}\right|_{v}, \cdots,\left|a_{n}\right|_{v}\right)=1$. Hence, applying the product formula for $b \in \mathcal{O}$, the above becomes

For $v \in S-S_{\infty}$,

$$
\prod_{v \in S}|b|_{v}^{a-\beta} \max \left(|b|_{v},\left|a_{1}\right|_{v}, \ldots,\left|a_{n}\right|_{v}\right)^{-a}
$$

$$
\operatorname{ord}_{p}(b)=\operatorname{ord}_{p}(b)+\operatorname{ord}_{p}\left(r_{j}\right), \quad \operatorname{ord}_{p}\left(a_{i}\right)=\operatorname{ord}_{p}\left(a_{i}\right)+\operatorname{ord}_{p}\left(r_{j}\right)
$$

whence $\max _{v \in S-S_{\infty}}\left(|b|_{v},\left|a_{1}\right|_{v}, \ldots,\left|a_{n}\right|_{v}\right)=N p^{-o r d_{p}\left(s_{j}\right)}$, since $b, a_{1}, \ldots, a_{n}$ are relatively prime. Here $N p=\operatorname{card}(\Theta / p)$. Setting $c_{p}=\max \left\{\operatorname{ord}_{p}\left(r_{j}\right), 1 \leq j \leq b\right\}$, $c^{\prime}\left(\Pi_{v \in S-S_{\infty}} N p^{c p}\right)^{a}$, we find

$$
\prod_{v} \max \left(1,\left|i_{1}\right|_{\nu}, \cdots,\left|i_{n}\right|_{\nu}\right)^{-\sigma} \leq c^{\prime}\left(\prod_{\nu \in S}|b|_{\nu}\right)^{a-\beta} \max \left(|b|_{\infty},\left|a_{1}\right|_{\infty}, \cdots,\left|a_{n}\right|_{\infty}\right)^{-a} \text {. }
$$

Therefore, it suffices to show that the sum on the right, for $\left(a_{1}, \ldots, a_{n}\right) \in \Theta^{n}$ and $C_{b}$ over the set of principal ideals, $\neq 0$ of $\mathcal{Q}$, is convergent.

Since $|b|_{\infty} \geq 1$ for $b \neq 0 \in \mathcal{C}$ and $a>n$, we can apply Lemma 2 repeatedly, to show

$$
\sum_{\left(a_{1}, \cdots, a_{n}\right) \in O^{n}} \max \left(|b|_{\infty},\left|a_{1}\right|_{\infty}, \cdots,\left|a_{n}\right|_{\infty}\right)^{-a} \leq c^{n}|b|_{\infty}^{n-a}
$$

where $c$ is a fixed constant, independent of $b$. Hence, it suffices to show that the series $\Sigma_{O b}\left(\Pi_{v \in S}|b|_{v}\right)^{a-\beta}|b|_{\infty}^{n-a}$ is convergent. By the product formula, this is

$$
\begin{aligned}
& \sum_{O b}\left(\prod_{v \in S-S_{\infty}}|b|_{v}\right)^{a-n}\left(\prod_{v \xi S}|b|_{v}\right)^{\beta-n} \\
& =\sum_{O b}\left(\prod_{p \in S-S_{\infty}}(N p)^{-o r d_{p}(b)}\right)^{a-n}\left(\prod_{p \notin S} N_{p}^{- \text {ord }_{p}(b)}\right)^{\beta-n} \\
& <\sum_{\eta \neq n, \text { all integral ideals }}\left(\prod_{p \in S-S_{\infty}} N_{p}^{- \text {ord } p(\eta)}\right)^{a-n}\left(\prod_{p \notin S} N_{p}^{- \text {ord }_{p}(\eta)}\right)^{\beta-n} .
\end{aligned}
$$


But, by the Euler product, this differs by only an elementary factor from $\Sigma_{q \in 0, \text { integral }}(N \mathscr{Y})^{-\sigma}$. But for $\sigma=\beta-n>1$ ' this is convergent.

5. The Siegel formula. The character $\chi$ of $k_{A}$ puts it into duality with $k_{A}^{*}$ by $\left(i, i^{*}\right) \mapsto \chi^{\left(i i^{*}\right)}$, for $i, i^{*} \in k_{A}$. Identifying $X_{A}$ with its dual by $(x, y) \mapsto$ $\chi\left(x^{t} y\right)$, for $x, y \in X_{A}$, the autodual measure $|d x|_{A}$ on $X_{A}$ is then the Haar measure for which $X_{A} / X_{k}$ has measure 1 .

For every $\Phi \in \mathcal{S}\left(X_{A}\right)$, define

$$
F_{\Phi}^{*}\left(i^{*}\right)=\int_{X_{A}} \Phi(x) \chi\left(q(x) i^{*}\right)|d x|_{A},
$$

for $i^{*} \in k_{A}$.

For almost all $v$, the usual Haar measure on $k_{v}$ is autodual, $\mathcal{O}_{v}$ is the kernel of $X_{v}$ and $m\left(X_{v}^{0}\right)=1$. Recall that $X_{A}$ is the inductive limit of $X_{s}=X_{0}^{0} \times X_{1}$, where $X_{0}^{0}=\Pi_{v \triangleleft S} X_{v}^{0}, X_{1}=\Pi_{v \in S} X_{v}$, for $S$ running over the family of finite sets of valuations on $k$. Therefore, for every compact subset $C$ of $\delta\left(X_{A}\right)$, there exist an $S$ and a compact subset $C_{1}$ of $\mathcal{S}\left(x_{1}\right)$, such that every $\Phi \in C$ is of the form $\Phi_{0} \otimes \Phi_{1}$, where $\Phi_{0}$ is the characteristic function of $x_{0}^{0}, \Phi_{1}$ is in $C_{1}$.

Put $\sigma_{v}=m / 2$ for all $v$. Then, by Lemma 1 and Fubini's theorem, there is a positive constant $c$ such that

$$
\sum_{i \in k}\left|F_{\Phi}^{*}\left(i^{*}\right)\right| \leq c \sum_{i \in k} \prod_{\nu} \max \left(1,\left|i^{*}\right|_{\nu}\right)^{-\sigma}
$$

for every $\Phi \in C$. By Proposition 1, the right-hand side is convergent for $m \geq 5$.

Also, the mapping

$$
\left(X_{A}\right) \times k_{A} \longrightarrow \delta\left(X_{A}\right)
$$

$\omega$

$\left(\Phi, i^{*}\right) \longmapsto \Phi_{i}^{*}$

where $\Phi_{i^{*}}(x)=\Phi(x) \chi\left(q(x) i^{*}\right)$ is continuous. Hence, by Weil's criterion [7, p. 8], the continuous mapping $q: X_{A} \rightarrow k_{A}$ satisfies "condition (A)" and the following Poisson formula:

$$
\sum_{i \in k}^{*} F_{\Phi}^{*}\left(i^{*}\right)=\sum_{i \in k}\left(F_{\phi}^{*}\right)^{*}(i)
$$

Here $\left(F_{\Phi}^{*}\right) *(i)=F_{\Phi}(-i)$ for every $i \in k_{A}$.

Lemma 3. $F_{\Phi}(i)=\int_{U(i)_{A}} \Phi\left|D_{i}\right|_{A}$, for every $i \in k_{A}$.

Proof. It suffices to show this for $\Phi$ restricted to a subset of $\mathcal{S}\left(x_{A}\right)$ which spans a dense subspace of $\mathcal{S}\left(X_{A}\right)$. Take $\Phi=\Pi_{v} \Phi_{v}$, where $\Phi_{v} \in \mathcal{S}\left(X_{v}\right)$ for every 
$v$ and $\Phi_{v}=$ the characteristic function of $X_{v}^{0}$, for all but finitely many $v$. Then $F_{\Phi}$ decomposes into the product of $F_{\Phi_{v}}$, defined by $F_{\Phi_{v}}\left(i_{\nu}\right)=\left(F_{\Phi_{v}}^{*}\right) *\left(-i_{v}\right)$, whence, by the results of $\S 3, F_{\Phi_{v}}\left(i_{v}\right)=\int_{U_{v}\left(i_{v}\right)} \Phi_{v} \cdot\left|D_{v_{0} i}\right|_{v}$, for every $i_{v} \in k_{v}$. This implies the desired result. Therefore, (4) now reads

$$
\sum_{i \in k}^{* *} \int_{X_{A}} \Phi(x) \chi\left(q(x)_{i}^{*}\right)|d x|_{A}=\sum_{i \in k} \int_{U(i)} \Phi\left|D_{i}\right|_{A^{*}}
$$

Combining this with (1) and the exceptional orbit $\{0\}$, we obtain the Siegel formula,

Theorem.

$$
\int_{G_{A} / G_{k}}\left(\sum_{\xi \in X_{k}} \Phi(g \cdot \xi)\right)|d g|_{A}=2 \sum_{i \in k} \int_{X_{A}} \Phi(x) \chi\left(q(x) i^{*}\right)|d x|_{A}+2 \Phi(0),
$$

which is valid for $m \geq 5$. Here $G$ is the special orthogonal group, acting on $X$, of dimension m.

\section{REFERENCES}

1. A. Borel and T. Springer, Rationality properties of linear algebraic groups, Algebraic Groups and Discontinuous Subgroups (Proc. Sympos. Pure Math., Boulder, Colo., 1965), Amer. Math. Soc., Providence, R. I., 1966, pp. 26-32. MR 34 \#5823.

2. J. Igusa, On certain representations of semi-simple algebraic groups and the arithmetic of the corresponding invariants. I, Invent. Math. 12 (1971), 62-94. MR 45 \#6823.

3. - On the arithmetic of Pfaffians, Nagoya Math. J. 47 (1972), 169-198.

4. A. Weil, Basic number theory, Die Grundlehren der math. Wissenschaften, Band 144, Springer-Verlag, New York, 1967. MR 38 \#3244. 1961.

5. - Adeles and algebraic groups, Inst. for Advanced Study, Princeton, N. J.,

6. - Sur certains groupes d'opérateurs unitaires, Acta Math. 111 (1964), 143211. MR $29 \# 2324$ 。

7. - Sur la formule de Siegel dans la théorie des groupes classiques, Acta Math. 113 (1965), 1-87. MR 36 \#6421.

DEPARTMENT OF MATHEMATICS, UNIVERSITY OF MARYLAND, COLLEGE PARK, MARYLAND 20742

Current address: Department of Mathematics, University of Washington, Seattle, Washington 98195 